\title{
Article
}

\section{Fixed Points of Proinov E-Contractions}

\author{
Maryam A. Alghamdi ${ }^{1}$, Selma Gulyaz-Ozyurt ${ }^{2}$ (I) and Andreea Fulga $3, * \mathbb{C}$ \\ 1 Department of Mathematics, College of Science, University of Jeddah, Jeddah 21589, Saudi Arabia; \\ maalghamdi@uj.edu.sa \\ 2 Department of Mathematics, Sivas Cumhuriyet University, Sivas 58140, Turkey; sgulyaz@cumhuriyet.edu.tr \\ or selmagulyaz@gmail.com \\ 3 Department of Mathematics and Computer Sciences, Transilvania University of Brasov, B-dul Eroilor nr.29, \\ 500036 Brasov, Romania \\ * Correspondence: afulga@unitbv.ro
}

check for

updates

Citation: Alghamdi, M.A.;

Gulyaz-Ozyurt S.; Fulga, A. Fixed Points of Proinov E-Contractions. Symmetry 2021, 13, 962. https:// doi.org/10.3390/sym13060962

Academic Editors: Olivia Ana Florea and Ileana Constanţa Roşca

Received: 04 May 2021

Accepted: 25 May 2021

Published: 28 May 2021

Publisher's Note: MDPI stays neutral with regard to jurisdictional claims in published maps and institutional affiliations.

Copyright: (c) 2021 by the authors. Licensee MDPI, Basel, Switzerland. This article is an open access article distributed under the terms and conditions of the Creative Commons Attribution (CC BY) license (https:/ / creativecommons.org/licenses/by/ $4.0 /)$.
Abstract: In this paper, we consider a new type of Proinov contraction on the setting of a symmetrical abstract structure, more precisely, the metric space. Our goal is to expand on some results from the literature using admissible mappings and the concept of E-contraction. The considered examples indicate the validity of the obtained results.

Keywords: Proinov type contraction; E-contraction; alpha-admissible

\section{Introduction and Preliminaries}

Fixed point theory is one of the most dynamic research topics of the last two decades. New and interesting results are obtained, following especially two directions: changing the frame (the structure of the abstract space-e.g., $b$-metric, delta symmetric quasi-metric or non-symmetric metric space, etc.) or changing the property of the operators.

The notion of E-contraction was introduced by Fulga and Proca [1]. Later, this concept has been improved by several authors, e.g., [2-4]. Undoubtedly, one of the most interesting, most original, most impressive fixed point theorem published in the last two decades is the result of Proinov [5]. By using certain auxiliary functions, Proinov [5] obtained interesting fixed point theorems that generalize, extend, and unify several recent fixed point results in the literature.

In this paper, we shall propose a new type of contraction, namely, Proinov type $E$-contraction, which combines the Proinov approach and the E-contraction setting.

First, we recall the basic results and definitions.

Definition 1. Let $(\mathrm{X}, \mathrm{d})$ be a metric space and the functions $\vartheta, \theta:(0, \infty) \rightarrow \mathbb{R}$. A mapping $\mathcal{T}: \mathrm{X} \rightarrow \mathrm{X}$ is said to be a Proinov type contraction if

$$
\vartheta(\mathrm{d}(\mathcal{T} \mathrm{x}, \mathcal{T} \mathrm{y})) \leq \theta(\mathrm{d}(\mathrm{x}, \mathrm{y})),
$$

for all $x, y \in \mathrm{X}$ with $\mathrm{d}(\mathcal{T} x, \mathcal{T} y)>0$.

Theorem 1 ([5]). Let $(\mathrm{X}, \mathrm{d})$ be a complete metric space and $\mathcal{T}: \mathrm{X} \rightarrow \mathrm{X}$ be a Proinov type contraction, where the functions $\vartheta, \theta:(0, \infty) \rightarrow \mathbb{R}$ are such that the following conditions are satisfied:

(1) $\vartheta$ is non-decreasing;

(2) $\theta(s)<\vartheta(s)$ for any $s>0$;

(3) $\limsup _{s \rightarrow s_{0}+} \theta(s)<\vartheta\left(s_{0}+\right)$ for any $s_{0}>0$,

then $\mathcal{T}$ admits a unique fixed point.

Definition 2. Let $(\mathrm{X}, \mathrm{d})$ be a metric space and the functions $\vartheta, \theta:(0, \infty) \rightarrow \mathbb{R}$. A mapping $\mathcal{T}: \mathrm{X} \rightarrow \mathrm{X}$ is said to be a generalized Proinov type contraction if 


$$
\vartheta(\mathrm{d}(\mathcal{T} \mathrm{x}, \mathcal{T} \mathrm{y})) \leq \theta\left(\max \left\{\mathrm{d}(\mathrm{x}, \mathrm{y}), \mathrm{d}(\mathrm{x}, \mathcal{T} \mathrm{x}), \mathrm{d}(\mathrm{y}, \mathcal{T} \mathrm{y}), \frac{\mathrm{d}(\mathrm{x}, \mathcal{T} \mathrm{y})+\mathrm{d}(\mathrm{y}, \mathcal{T} \mathrm{x})}{2}\right\}\right)
$$

for all $x, y \in \mathrm{X}$ with $\mathrm{d}(\mathcal{T} x, \mathcal{T} y)>0$.

Theorem 2 ([5]). Let $(\mathrm{X}, \mathrm{d})$ be a complete metric space and $\mathcal{T}: \mathrm{X} \rightarrow \mathrm{X}$ be a generalized Proinov type contraction, where the functions $\vartheta, \theta:(0, \infty) \rightarrow \mathbb{R}$ are such that the following conditions are satisfied:

(1) $\vartheta$ is non-decreasing and $\theta(s)<\vartheta(s)$ for any $s>0$;

(2) $\theta\left(s_{0}\right)<\lim _{s \rightarrow s_{0}} \vartheta(s)$, for any $s_{0}>0$;

(3) $\limsup _{s \rightarrow s_{0}+} \theta(s)<\vartheta\left(s_{0}+\right)$ for any $s_{0}>0$.

Then $\mathcal{T}$ admits a unique fixed point.

Remark 1. Notice that in [5], the author did not put the completeness assumption of the metric space; but, he used in the proof.

Lemma 1 ([5]). Let $\left\{\mathrm{x}_{m}\right\}$ be a sequence in a metric space $(\mathrm{X}, \mathrm{d})$ such that $\mathrm{d}\left(\mathrm{x}_{m}, \mathrm{x}_{m+1}\right) \rightarrow 0$ as $m \rightarrow \infty$. If the sequence $\left\{x_{m}\right\}$ is not Cauchy, then there exist $e>0$ and the subsequences $\left\{m_{i}\right\}$ and $\left\{p_{i}\right\}$ of positive integers such that

$$
\begin{aligned}
\lim _{i \rightarrow \infty} \mathrm{d}\left(\mathrm{x}_{m_{i}}, \mathrm{x}_{p_{i}}\right) & =\lim _{i \rightarrow \infty} \mathrm{d}\left(\mathrm{x}_{m_{i}+1}, \mathrm{x}_{p_{i}}\right)=\lim _{i \rightarrow \infty} \mathrm{d}\left(\mathrm{x}_{m_{i}}, \mathrm{x}_{p_{i}+1}\right) \\
& =\lim _{i \rightarrow \infty} \mathrm{d}\left(\mathrm{x}_{m_{i}+1}, \mathrm{x}_{p_{i}+1}\right)=e
\end{aligned}
$$

In 2012, Karapinar-Samet proposed [6] generalized $\alpha-\psi$-contraction. In this consideration, the role of $\alpha$ admissible mapping is to combine the problem of fixed point of "cyclic contractions" with the same problem in the framework of metric spaces endowed with a"partially ordered set", see, e.g., [7-12]. Later, this notion is refined by Popescu [13]. Let $(X, d)$ be a metric space, $\mathcal{T}: X \rightarrow X$ be a mapping and a given mapping $\alpha: X \times X \rightarrow[0, \infty)$. We say that $\mathcal{T}$ is triangular $\alpha$-orbital admissible (on short $\alpha$-t.o.a.) [13] if the following two conditions are satisfied

(o) $\alpha(\mathrm{x}, \mathcal{T} \mathrm{x}) \geq 1 \Rightarrow \alpha\left(\mathcal{T} \mathrm{x}, \mathcal{T}^{2} \mathrm{x}\right) \geq 1$ for any $\mathrm{x} \in \mathrm{X}$;

$\left(t_{o}\right) \quad \alpha(\mathrm{x}, \mathrm{y}) \geq 1$ and $\alpha(\mathrm{y}, \mathcal{T} \mathrm{y}) \geq 1 \Rightarrow \alpha(\mathrm{x}, \mathcal{T} \mathrm{y}) \geq 1$ for any $\mathrm{x}, \mathrm{y} \in \mathrm{X}$.

Lemma 2 ([13]). Let $\left\{\mathrm{x}_{m}\right\}$ be a sequence on a non-empty set $\mathrm{X}$ defined by $\mathrm{x}_{m}=\mathcal{T} \mathrm{x}_{m-1}$ for any $m \in \mathbb{N}$, where $\mathcal{T}: \mathbf{X} \rightarrow \mathbf{X}$ is an $\alpha$-t.o.a.mapping. If there exists $\mathrm{x}_{0} \in \mathbf{X}$ such that $\alpha\left(\mathrm{x}_{0}, \mathcal{T} \mathrm{x}_{0}\right) \geq 1$, then $\alpha\left(\mathrm{x}_{n}, \mathrm{x}_{m}\right) \geq 1$, for all $n, m \in \mathbb{N}$.

\section{Main Results}

Throughout this section, we will consider that $\vartheta, \theta:(0, \infty) \rightarrow \mathbb{R}$ are two functions such that

$\left(m_{0}\right) \theta(s)<\vartheta(s)$, for all $s>0$

and $\alpha: \mathrm{X} \times \mathrm{X} \rightarrow[0,+\infty)$

Definition 3. A mapping $\mathcal{T}: \mathrm{X} \rightarrow \mathrm{X}$ on a complete metric space $(\mathrm{X}, \mathrm{d})$ is $a(\alpha, \vartheta, \theta)$ - $\mathcal{E}$ contraction if the inequality

$$
\alpha(\mathrm{x}, \mathrm{y}) \vartheta(\mathrm{d}(\mathcal{T} \mathrm{x}, \mathcal{T} \mathrm{y})) \leq \theta(\mathcal{E}(\mathrm{x}, \mathrm{y})),
$$

holds for every distinct $\mathrm{x}, \mathrm{y} \in \mathrm{X}$ such that $\mathrm{d}(\mathcal{T} \mathrm{x}, \mathcal{T} \mathrm{y})>0$, where

$$
\mathcal{E}(\mathrm{x}, \mathrm{y})=\max \left\{\mathrm{d}(\mathrm{x}, \mathrm{y})+|\mathrm{d}(\mathrm{x}, \mathcal{T} \mathrm{x})-\mathrm{d}(\mathrm{y}, \mathcal{T} \mathrm{y})|, \frac{\mathrm{d}(\mathrm{x}, \mathcal{T} \mathrm{y})+\mathrm{d}(\mathrm{y}, \mathcal{T} \mathrm{x})}{2}\right\}
$$


Theorem 3. Let $(\mathrm{X}, \mathrm{d})$ be a complete metric space and $\mathcal{T}: \mathrm{X} \rightarrow \mathrm{X}$ be $a(\alpha, \vartheta, \theta)$-E contraction such that:

$\left(m_{1}\right) \vartheta$ is lower semi-continuous and non-decreasing;

$\left(m_{2}\right) \limsup _{s \rightarrow s_{0}} \theta(s)<\vartheta\left(s_{0}\right)$ for any $s_{0}>0$;

$\left(m_{3}\right) \mathcal{T}$ is $\alpha$-t.o.a. and there exists $\mathrm{x}_{0} \in \mathrm{X}$ such that $\alpha\left(\mathrm{x}_{0}, \mathcal{T} \mathrm{x}_{0}\right) \geq 1$;

$\left(m_{4}\right) \alpha\left(x_{m}, x_{*}\right) \geq 1$ for any sequence $\left\{x_{m}\right\}$ such that $x_{m} \rightarrow x_{*}$ and $\alpha\left(x_{m}, x_{m+1}\right) \geq 1$, for any $m \in \mathbb{N}$.

Then, $\mathcal{T}$ possesses a fixed point.

Proof. Let $\left\{\mathrm{x}_{m}\right\}$ be the sequence in $\mathrm{X}$ defined as $\mathrm{x}_{1}=\mathcal{T} \mathrm{x}_{0}, \mathrm{x}_{m}=\mathcal{T} \mathrm{x}_{m-1}$, for any $m \in \mathbb{N}$, where $x_{0}$ is an arbitrary fixed point in $X$ such that $\alpha\left(x_{0}, \mathcal{T} x_{0}\right) \geq 1$. We can assume that $\mathrm{d}\left(\mathrm{x}_{m}, \mathrm{x}_{m+1}\right)>0$ for every $m \in \mathbb{N}$, because, on the contrary, if we can find $m_{l} \in \mathbb{N}$ such that $x_{m_{l}}=x_{m_{l}+1}$, due to the definition of the sequence $\left\{x_{m_{l}}\right\}$ we have $d\left(\mathcal{T} x_{m_{l}}, x_{m_{l}}\right)=$ $\mathrm{d}\left(\mathrm{x}_{m_{l}+1}, \mathrm{x}_{m_{l}}\right)=0$, which means $\mathcal{T} \mathrm{x}_{m_{l}}=\mathrm{x}_{m_{l}}$. Therefore, letting $\mathrm{x}=\mathrm{x}_{m}$ and $\mathrm{y}=\mathrm{x}_{m+1}$ in (4), using $\left(m_{0}\right)$ and taking Lemma 2 into account, we have

$$
\begin{aligned}
\vartheta\left(\mathrm{d}\left(\mathrm{x}_{m+1}, \mathrm{x}_{m+2}\right)\right. & =\vartheta\left(\mathrm{d}\left(\mathcal{T} \mathrm{x}_{m}, \mathcal{T} \mathrm{x}_{m+1}\right)\right) \leq \alpha\left(\mathrm{x}_{m}, \mathrm{x}_{m+1}\right) \vartheta\left(\mathrm{d}\left(\mathcal{T} \mathrm{x}_{m}, \mathcal{T} \mathrm{x}_{m+1}\right)\right) \\
& \leq \theta\left(\mathcal{E}\left(\mathrm{x}_{m}, \mathrm{x}_{m+1}\right)\right)<\vartheta\left(\mathcal{E}\left(\mathrm{x}_{m}, \mathrm{x}_{m+1}\right)\right)
\end{aligned}
$$

where

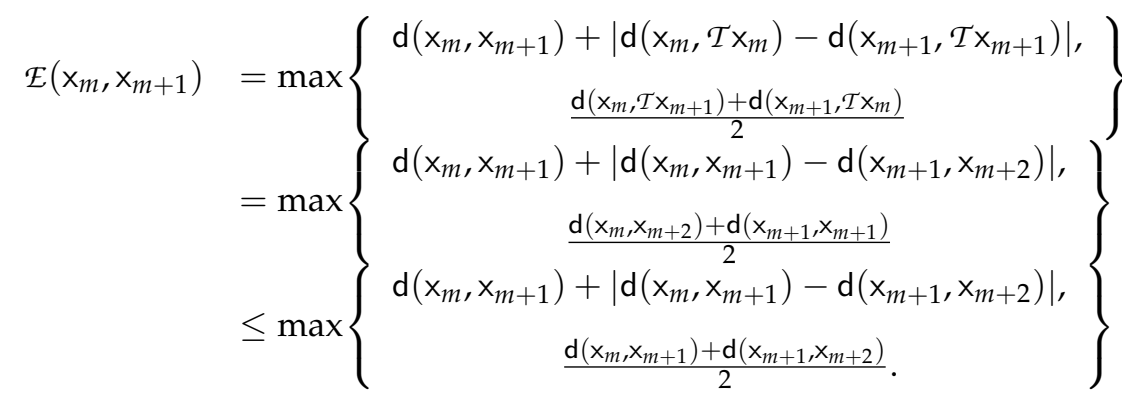

$$
\begin{aligned}
& \text { If } \max \left\{\mathrm{d}\left(\mathrm{x}_{m}, \mathrm{x}_{m+1}\right), \mathrm{d}\left(\mathrm{x}_{m+1}, \mathrm{x}_{m+2}\right)\right\}=\mathrm{d}\left(\mathrm{x}_{m+1}, \mathrm{x}_{m+2}\right) \text {, } \\
& \mathcal{E}\left(\mathrm{x}_{m}, \mathrm{x}_{m+1}\right) \leq \max \left\{\mathrm{d}\left(\mathrm{x}_{m+1}, \mathrm{x}_{m+2}\right), \mathrm{d}\left(\mathrm{x}_{m+1}, \mathrm{x}_{m+2}\right)\right\}=\mathrm{d}\left(\mathrm{x}_{m+1}, \mathrm{x}_{m+2}\right)
\end{aligned}
$$

and the inequality (6) yields

$$
\vartheta\left(\mathrm{d}\left(\mathrm{x}_{m+1}, \mathrm{x}_{m+2}\right)\right) \leq \theta\left(\mathcal{E}\left(\mathrm{x}_{m}, \mathrm{x}_{m+1}\right)\right)<\vartheta\left(\mathcal{E}\left(\mathrm{x}_{m}, \mathrm{x}_{m+1}\right)\right)<\vartheta\left(\mathrm{d}\left(\mathrm{x}_{m+1}, \mathrm{x}_{m+2}\right)\right),
$$

which is a contradiction.

$$
\begin{aligned}
& \text { If } \max \left\{\mathrm{d}\left(\mathrm{x}_{m}, \mathrm{x}_{m+1}\right), \mathrm{d}\left(\mathrm{x}_{m+1}, \mathrm{x}_{m+2}\right)\right\}=\mathrm{d}\left(\mathrm{x}_{m}, \mathrm{x}_{m+1}\right), \\
& \qquad \begin{aligned}
\mathcal{E}\left(\mathrm{x}_{m}, \mathrm{x}_{m+1}\right) \leq & \leq \max \left\{2 \mathrm{~d}\left(\mathrm{x}_{m}, \mathrm{x}_{m+1}\right)-\mathrm{d}\left(\mathrm{x}_{m+1}, \mathrm{x}_{m+2}\right), \mathrm{d}\left(\mathrm{x}_{m}, \mathrm{x}_{m+1}\right)\right\} \\
& =2 \mathrm{~d}\left(\mathrm{x}_{m}, \mathrm{x}_{m+1}\right)-\mathrm{d}\left(\mathrm{x}_{m+1}, \mathrm{x}_{m+2}\right)
\end{aligned}
\end{aligned}
$$

and the inequality (6) becomes

$$
\begin{aligned}
\vartheta\left(\mathrm{d}\left(\mathrm{x}_{m+1}, \mathrm{x}_{m+2}\right)\right) & \leq \theta\left(\mathcal{E}\left(\mathrm{x}_{m}, \mathrm{x}_{m+1}\right)\right)<\vartheta\left(\mathcal{E}\left(\mathrm{x}_{m}, \mathrm{x}_{m+1}\right)\right) \\
& <\vartheta\left(2 \mathrm{~d}\left(\mathrm{x}_{m}, \mathrm{x}_{m+1}\right)-\mathrm{d}\left(\mathrm{x}_{m+1}, \mathrm{x}_{m+2}\right)\right)
\end{aligned}
$$

Therefore, by $\left(m_{1}\right)$, we get

$$
\mathrm{d}\left(\mathrm{x}_{m+1}, \mathrm{x}_{m+2}\right)<2 \mathrm{~d}\left(\mathrm{x}_{m}, \mathrm{x}_{m+1}\right)-\mathrm{d}\left(\mathrm{x}_{m+1}, \mathrm{x}_{m+2}\right) \Rightarrow \mathrm{d}\left(\mathrm{x}_{m+1}, \mathrm{x}_{m+2}\right)<\mathrm{d}\left(\mathrm{x}_{m}, \mathrm{x}_{m+1}\right),
$$


so that, the sequence $\left\{d\left(x_{m}, x_{m+1}\right)\right\}$ is a decreasing and bounded below by 0 . Thus, we can find $\delta \geq 0$, such that $\lim _{m \rightarrow \infty} \mathrm{d}\left(\mathrm{x}_{m}, \mathrm{x}_{m+1}\right)=\delta$. Thus, $\lim _{m \rightarrow \infty} \mathcal{E}\left(\mathrm{x}_{m}, \mathrm{x}_{m+1}\right)=\delta$ and we claim that, in fact, $\delta=0$. Supposing on the contrary, that $\delta>0$, letting $m \rightarrow \infty$ in the first part of (6), we have

$$
\vartheta(\delta)=\lim _{m \rightarrow \infty} \vartheta\left(\mathrm{d}\left(\mathrm{x}_{m+1}, \mathrm{x}_{m+2}\right)\right) \leq \limsup _{m \rightarrow \infty} \theta\left(\mathcal{E}\left(\mathrm{x}_{m}, \mathrm{x}_{m+1}\right)\right) \leq \limsup _{s \rightarrow \delta} \vartheta(s),
$$

but this contradicts the assumption $\left(m_{2}\right)$. Consequently, $\delta=0$, that is

$$
\lim _{m \rightarrow \infty} \mathrm{d}\left(\mathrm{x}_{m}, \mathrm{x}_{m+1}\right)=\lim _{m \rightarrow \infty} \mathrm{d}\left(\mathrm{x}_{m}, \mathcal{T} \mathrm{x}_{m}\right)=0 \text {. }
$$

Supposing that $\left\{x_{n}\right\}$ is not a Cauchy sequence, from Lemma 1, we can find two subsequences $\left\{x_{m_{i}}\right\},\left\{x_{p_{i}}\right\}$ of the sequence $\left\{x_{m}\right\}$ such that (3) hold. Thus, setting $u_{i}=$ $\mathrm{d}\left(\mathrm{x}_{m_{i}}, \mathrm{x}_{p_{i}}\right)$, respectively $v_{i}=\mathcal{E}\left(\mathrm{x}_{m_{i}}, \mathrm{x}_{p_{i}}\right)$, and taking (10), (3) into account, we have

$$
\begin{aligned}
\lim _{i \rightarrow \infty} u_{i} & =e, \\
\lim _{i \rightarrow \infty} v_{i} & =\lim _{i \rightarrow \infty} \mathcal{E}\left(\mathrm{x}_{m_{i}}, \mathrm{x}_{p_{i}}\right)=\lim _{i \rightarrow \infty} \max \left\{\begin{array}{c}
\left(\mathrm{d}\left(\mathrm{x}_{m_{i}}, \mathrm{x}_{p_{i}}\right)+\left|\mathrm{d}\left(\mathrm{x}_{m_{i}}, \mathcal{T} \mathrm{x}_{m_{i}}\right)-\mathrm{d}\left(\mathrm{x}_{p_{i}}, \mathcal{T} \mathrm{x}_{p_{i}}\right)\right|,\right. \\
\frac{\mathrm{d}\left(\mathrm{x}_{m_{i}}, \mathcal{T} \mathrm{x}_{p_{i}}\right)+\mathrm{d}\left(\mathrm{x}_{p_{i}}, \mathcal{T} \mathrm{x}_{m_{i}}\right)}{2}
\end{array}\right\} \\
& =\lim _{i \rightarrow \infty} \max \left\{\begin{array}{c}
\left(\mathrm{d}\left(\mathrm{x}_{m_{i}}, \mathrm{x}_{p_{i}}\right)+\left|\mathrm{d}\left(\mathrm{x}_{m_{i}}, \mathrm{x}_{m_{i}+1}\right)-\mathrm{d}\left(\mathrm{x}_{p_{i}}, \mathrm{x}_{p_{i}+1}\right)\right|,\right. \\
\frac{\mathrm{d}\left(\mathrm{x}_{m_{i}}, \mathrm{x}_{p_{i}+1}\right)+\mathrm{d}\left(\mathrm{x}_{p_{i}}, \mathrm{x}_{m_{i}+1}\right)}{2}
\end{array}\right\} \\
& =e .
\end{aligned}
$$

Therefore, applying (4) for $\mathrm{x}=\mathrm{x}_{m_{i}}$ and $\mathrm{y}=\mathrm{x}_{p_{i}}$, and using Lemma 2, we get

$$
\vartheta\left(u_{i+1}\right)=\vartheta\left(\mathrm{d}\left(\mathrm{x}_{m_{i}+1}, \mathrm{x}_{p_{i}+1}\right)\right) \leq \alpha\left(\mathrm{x}_{m_{i}}, \mathrm{x}_{p_{i}}\right) \vartheta\left(\mathrm{d}\left(\mathcal{T} \mathrm{x}_{m_{i}}, \mathcal{T} \mathrm{x}_{p_{i}}\right)\right) \leq \theta\left(\mathcal{E}\left(\left(\mathrm{x}_{m_{i}}, \mathrm{x}_{p_{i}}\right)\right)=\theta\left(v_{i}\right) .\right.
$$

Thereupon, considering the limit superior in the above inequality, we have

$$
\vartheta(e)=\lim _{i \rightarrow \infty} \vartheta\left(u_{i}\right) \leq \limsup _{i \rightarrow \infty} \theta\left(v_{i}\right) \leq \limsup _{s \rightarrow e} \theta(s),
$$

which contradicts $\left(m_{2}\right)$. Consequently, $\left\{x_{m}\right\}$ is a Cauchy sequence on a complete metric space, which guarantees that $\left\{x_{m}\right\}$ is a convergent sequence. Denoting by $x_{*}$ the limit of this sequence, we will show that under the assumption $\left(m_{4}\right)$ this point is a fixed point for the mapping $\mathcal{T}$. Indeed, if not, then we have

$$
0<\mathrm{d}\left(\mathrm{x}_{*}, \mathcal{T} \mathrm{x}_{*}\right) \leq \mathrm{d}\left(\mathrm{x}_{*}, \mathcal{T} \mathrm{x}_{m}\right)+\mathrm{d}\left(\mathcal{T} \mathrm{x}_{m}, \mathcal{T} \mathrm{x}_{*}\right)
$$

If $\mathrm{d}\left(\mathcal{T} \mathrm{x}_{m}, \mathcal{T} \mathrm{x}_{*}\right)=0$ for infinitely many values of $m$, then (12) becomes $0<\mathrm{d}\left(\mathrm{x}_{*}, \mathcal{T} \mathrm{X}_{*}\right) \leq$ $\mathrm{d}\left(\mathrm{x}_{*}, \mathrm{x}_{m+1}\right) \rightarrow 0$ as $m \rightarrow \infty$, so, $\mathrm{d}\left(\mathrm{x}_{*}, \mathcal{T} \mathrm{x}_{*}\right)=0$.

If $\mathrm{d}\left(\mathcal{T} \mathrm{x}_{m}, \mathcal{T} \mathrm{x}_{*}\right)>0$, for any $m \in \mathbb{N}$, the relation (4) becomes

$$
\vartheta\left(\mathrm{d}\left(\mathrm{x}_{m+1}, \mathcal{T} \mathrm{x}_{*}\right)\right) \leq \alpha\left(\mathrm{x}_{m}, \mathrm{x}_{*}\right) \vartheta\left(\mathrm{d}\left(\mathcal{T} \mathrm{x}_{m}, \mathcal{T} \mathrm{x}_{*}\right)\right) \leq \theta\left(\mathcal{E}\left(\mathrm{x}_{m}, \mathrm{x}_{*}\right)\right)<\vartheta\left(\mathcal{E}\left(\mathrm{x}_{m}, \mathrm{x}_{*}\right)\right)
$$

(here, we used $\left(m_{2}\right)$ ), where

$$
\begin{aligned}
\mathcal{E}\left(\mathrm{x}_{m}, \mathrm{x}_{*}\right) & =\max \left\{\mathrm{d}\left(\mathrm{x}_{m}, \mathrm{x}_{*}\right)+\left|\mathrm{d}\left(\mathrm{x}_{m}, \mathcal{T} \mathrm{x}_{m}\right)-\mathrm{d}\left(\mathrm{x}_{*}, \mathcal{T} \mathrm{x}_{*}\right)\right|, \frac{\mathrm{d}\left(\mathrm{x}_{m}, \mathcal{T} \mathrm{x}_{*}\right)+\mathrm{d}\left(\mathrm{x}_{*}, \mathcal{T} \mathrm{x}_{m}\right)}{2}\right\} \\
& =\max \left\{\mathrm{d}\left(\mathrm{x}_{m}, \mathrm{x}_{*}\right)+\left|\mathrm{d}\left(\mathrm{x}_{m}, \mathrm{x}_{m+1}\right)-\mathrm{d}\left(\mathrm{x}_{*}, \mathcal{T} \mathrm{x}_{*}\right)\right|, \frac{\mathrm{d}\left(\mathrm{x}_{m}, \mathcal{T} \mathrm{x}_{*}\right)+\mathrm{d}\left(\mathrm{x}_{*}, \mathrm{x}_{m+1}\right)}{2}\right\} \\
& =\mathrm{d}\left(\mathrm{x}_{*}, \mathcal{T} \mathrm{x}_{*}\right),
\end{aligned}
$$


for $m$ sufficiently large. (Here, we took into account $\mathrm{d}\left(\mathrm{x}_{m}, \mathrm{x}_{*}\right) \rightarrow 0$ and $\mathrm{d}\left(\mathrm{x}_{m}, \mathrm{x}_{m+1}\right) \rightarrow 0$ as $m \rightarrow \infty$.) Thus, letting $m \rightarrow \infty$ in (13) and keeping in mind the lower-semicontinuity of $\vartheta$, we obtain

$\liminf _{s \rightarrow \mathrm{d}\left(\mathcal{T} \mathrm{x}_{*}, \mathrm{x}_{*}\right)} \vartheta(s) \leq \lim _{m \rightarrow \infty} \vartheta\left(\mathrm{d}\left(\mathcal{T} \mathrm{x}_{*}, \mathcal{T} \mathrm{x}_{m}\right)\right) \leq \theta\left(\mathrm{d}\left(\mathrm{x}_{*}, \mathcal{T} \mathrm{x}_{*}\right)\right)<\vartheta\left(\mathrm{d}\left(\mathrm{x}_{*}, \mathcal{T} \mathrm{x}_{*}\right)\right)<\liminf _{s \rightarrow \mathrm{d}\left(\mathcal{T} \mathrm{x}_{*}, \mathrm{x}_{*}\right)} \vartheta(s)$,

which is a contradiction. Then, $\mathrm{x}_{*}=\mathcal{T} \mathrm{x}_{*}$.

Theorem 4. Adding the condition

$\left(m_{5}\right) \alpha(\mathrm{v}, \mathrm{u}) \geq 1$, for any $\mathrm{v}, \mathrm{u} \in\{\mathrm{x} \in \mathrm{X}: \mathcal{T} \mathrm{x}=\mathrm{x}\}$

to the hypotheses of Theorem 3, one obtains uniqueness of the fixed point.

Proof. Supposing that $\mathrm{y}_{*} \in \mathrm{X}$ is such that $\mathcal{T} \mathrm{y}_{*}=\mathrm{y}_{*} \neq \mathrm{x}_{*}$, by (4) we have

$$
\begin{aligned}
\vartheta\left(\mathrm{d}\left(\mathrm{x}_{*}, \mathrm{y}_{*}\right)\right) & \leq \alpha\left(\mathrm{x}_{*}, \mathrm{y}_{*}\right) \vartheta\left(\mathrm{d}\left(\mathcal{T} \mathrm{x}_{*}, \mathcal{T} \mathrm{y}_{*}\right)\right) \leq \theta\left(\mathcal{E}\left(\mathrm{x}_{*}, \mathrm{y}_{*}\right)\right)<\vartheta\left(\mathcal{E}\left(\mathrm{x}_{*}, \mathrm{y}_{*}\right)\right) \\
& =\vartheta\left(\max \left\{\mathrm{d}\left(\mathrm{x}_{*}, \mathrm{y}_{*}\right)+\left|\mathrm{d}\left(\mathrm{x}_{*}, \mathcal{T} \mathrm{x}_{*}\right)-\mathrm{d}\left(\mathrm{y}_{*}, \mathcal{T} \mathrm{y}_{*}\right)\right|, \frac{\mathrm{d}\left(\mathrm{x}_{*}, \mathcal{T} \mathrm{y}_{*}\right)+\mathrm{d}\left(\mathrm{y}_{*}, \mathcal{T} \mathrm{x}_{*}\right)}{2}\right\}\right) \\
& =\vartheta\left(\max \left\{\mathrm{d}\left(\mathrm{x}_{*}, \mathrm{y}_{*}\right)+\left|\mathrm{d}\left(\mathrm{x}_{*}, \mathrm{x}_{*}\right)-\mathrm{d}\left(\mathrm{y}_{*}, \mathrm{y}_{*}\right)\right|, \frac{\mathrm{d}\left(\mathrm{x}_{*}, \mathrm{y}_{*}\right)+\mathrm{d}\left(\mathrm{y}_{*}, \mathrm{x}_{*}\right)}{2}\right\}\right) \\
& =\vartheta\left(\mathrm{d}\left(\mathrm{x}_{*}, \mathrm{y}_{*}\right)\right)
\end{aligned}
$$

which is a contradiction. Therefore, $x_{*}=y_{*}$.

Example 1. Let the set $\left.\mathrm{X}=\left\{O, A_{i}, B, C\right)\right\}, i \in\{0,1,2, \ldots, 7\}$, where $O=(0,0), B=(0,3)$, $C=\left(2, \frac{3}{2}\right)$ and $A_{i}$ with $i \in\{0,1, \ldots, 7\}$ are the corners of the regular octagon with a circumradius of $R=4$. Let $\mathrm{d}: \mathrm{X} \rightarrow \mathrm{X} \rightarrow[0,+\infty)$, where $\left.\mathrm{d}\left(U_{1}, U_{2}\right)\right)=\sqrt{\left(\mathrm{x}_{1}-\mathrm{x}_{2}\right)^{2}+\left(\mathrm{y}_{1}-\mathrm{y}_{2}\right)^{2}}$ for any $U_{1}, U_{2} \in \mathrm{X}, U_{1}=\left(\mathrm{x}_{1}, \mathrm{y}_{1}\right), U_{2}=\left(\mathrm{x}_{2}, \mathrm{y}_{2}\right)$. Let, also, the mapping $\mathcal{T}: \mathrm{X} \rightarrow \mathrm{X}$ be defined as

$$
\begin{aligned}
& \mathcal{T} O=B, \mathcal{T} B=C, \mathcal{T} C=O, \\
& \mathcal{T} A_{0}=A_{0}=\mathcal{T} A_{7}, \mathcal{T} A_{i}=A_{i+1}, \text { for any } i \in\{1,2, \ldots, 6\}
\end{aligned}
$$

First of all, we can remark that neither Theorem 1, nor Theorem 2 cannot be applied, because letting, for example, $\mathrm{x}=A$ and $\mathrm{y}=C$, we have

$$
\begin{aligned}
& \mathrm{d}(A, C)=\frac{5}{2}, \mathrm{~d}(\mathcal{T} A, \mathcal{T} C)=\mathrm{d}((4,0),(0,0))=4, \mathrm{~d}(A, \mathcal{T} A)=\mathrm{d}((4,0),(4,0))=0, \\
& \mathrm{~d}(C, \mathcal{T} C)=\mathrm{d}\left(\left(2, \frac{3}{2}\right),(0,0)\right)=\frac{5}{2}, \mathrm{~d}(A, \mathcal{T} C)=\mathrm{d}((4,0),(0,0))=4, \\
& \mathrm{~d}(\mathcal{T} A, C)=\mathrm{d}\left((4,0),\left(2, \frac{3}{2}\right)\right)=\frac{5}{2} .
\end{aligned}
$$

Thus, replacing in (1) and (2) we are leading to some contradictions, since

$$
\vartheta(4)=\vartheta(\mathrm{d}(\mathcal{T} A, \mathcal{T} C)) \leq \theta(\mathrm{d}(A, C))=\theta\left(\frac{5}{2}\right)<\vartheta\left(\frac{5}{2}\right)
$$

and

$$
\begin{aligned}
\vartheta(4) & =\vartheta(\mathrm{d}(\mathcal{T} A, \mathcal{T} C)) \leq \theta\left(\max \left\{\mathrm{d}(A, C), \mathrm{d}(A, \mathcal{T} A), \mathrm{d}(C, \mathcal{T} C), \frac{\mathrm{d}(A, \mathcal{T} C)+\mathrm{d}(C, \mathcal{T} A)}{2}\right\}\right) \\
& =\theta\left(\frac{13}{4}\right)<\vartheta\left(\frac{13}{4}\right) .
\end{aligned}
$$


Now, we consider the following functions:

$$
\begin{array}{ll}
\alpha: X \times X \rightarrow[0,+\infty), \alpha\left(U_{1}, U_{2}\right)=\left\{\begin{aligned}
1, & \text { for }\left(U_{1}, U_{2}\right) \in \mathcal{A} \cup \mathcal{B} \\
\frac{\mathrm{d}\left(U_{1}, U_{2}\right)}{2}, & \text { for }\left(U_{1}, U_{2}\right) \in \mathcal{C} \\
0, & \text { otherwise }
\end{aligned}\right. \\
\vartheta, \theta:(0, \infty) \rightarrow \mathbb{R}, \vartheta(s)=\frac{15}{16} s \text { and } \theta(s)=\frac{14}{16} s, &
\end{array}
$$

where $\mathcal{A}=\{(B, O),(A, O),(O, A),(B, A),(A, B)\}, \mathcal{B}=\left\{\left(A_{0}, A_{i}\right),\left(A_{i}, A_{0}\right) \mid i \in\{0,1, \ldots, 5,6\}\right\}$ and $C=\left\{\left(A_{0}, A_{7}\right),\left(A_{7}, A_{0}\right) \mid\right\}$. We can easily see that the assumptions $\left(m_{1}\right)-\left(m_{5}\right)$ hold. Thus, we have to prove that the mapping $\mathcal{T}$ is an $(\alpha, \vartheta, \theta)$-E contraction. So, consider the following cases:

- $U_{1}=O, U_{2}=A$,

$$
\begin{aligned}
\mathrm{d}(O, A) & =4, \mathrm{~d}(\mathcal{T} O, \mathcal{T} A)=5, \mathrm{~d}(A, \mathcal{T} A)=0, \mathrm{~d}(O, \mathcal{T} O)=3, \\
\mathrm{~d}(O, \mathcal{T} A) & =4, \mathrm{~d}(A, \mathcal{T} O)=5, \mathcal{E}(O, A)=7,
\end{aligned}
$$

and

$$
\alpha(O, A) \vartheta(\mathrm{d}(\mathcal{T} O, \mathcal{T} A))=\frac{15}{16} \cdot 5<\frac{14}{16} \cdot 7=\theta(\mathcal{E}(O, A)) .
$$

- $U_{1}=B, U_{2}=O$,

$$
\begin{aligned}
\mathrm{d}(B, O) & =3, \mathrm{~d}(\mathcal{T} B, \mathcal{T} O)=\frac{5}{2}, \mathrm{~d}(B, \mathcal{T} B)=\frac{5}{2}, \mathrm{~d}(O, \mathcal{T} O)=3, \\
\mathrm{~d}(B, \mathcal{T} O) & =0, \mathrm{~d}(O, \mathcal{T} B)=\frac{5}{2}, \mathcal{E}(B, O)=\frac{7}{2}
\end{aligned}
$$

and

$$
\alpha(B, O) \vartheta(\mathrm{d}(\mathcal{T} B, \mathcal{T} O))=\frac{15}{16} \cdot \frac{5}{2}<\frac{14}{16} \cdot \frac{7}{2}=\theta(\mathcal{E}(B, O)) .
$$

- $U_{1}=B, U_{2}=A$,

$$
\begin{aligned}
\mathrm{d}(B, A) & =5, \mathrm{~d}(\mathcal{T} B, \mathcal{T} A)=\frac{5}{2}, \mathrm{~d}(B, \mathcal{T} B)=\frac{5}{2}, \mathrm{~d}(A, \mathcal{T} A)=0, \\
\mathrm{~d}(B, \mathcal{T} A) & =5, \mathrm{~d}(A, \mathcal{T} B)=\frac{5}{2}, \mathcal{E}(B, A)=\frac{15}{2}
\end{aligned}
$$

and

$$
\alpha(B, A) \vartheta(\mathrm{d}(\mathcal{T} B, \mathcal{T} A))=\frac{15}{16} \cdot \frac{5}{2}<\frac{14}{16} \cdot \frac{15}{2}=\theta(\mathcal{E}(B, A)) .
$$

- $U_{1}=A, U_{2}=C$,

$$
\begin{aligned}
\mathrm{d}(A, C) & =\frac{5}{2}, \mathrm{~d}(\mathcal{T} A, \mathcal{T} C)=4, \mathrm{~d}(C, \mathcal{T} C)=\frac{5}{2}, \mathrm{~d}(A, \mathcal{T} A)=0, \\
\mathrm{~d}(C, \mathcal{T} A) & =\frac{5}{2}, \mathrm{~d}(A, \mathcal{T} C)=4, \mathcal{E}(A, C)=5,
\end{aligned}
$$

and

$$
\alpha(A, C) \vartheta(\mathrm{d}(\mathcal{T} A, \mathcal{T} C))=\frac{15}{16} \cdot 4<\frac{14}{16} \cdot 5=\theta(\mathcal{E}(A, C)) .
$$

- $U_{1}=A_{0}, U_{2}=A_{i}$ 


\begin{tabular}{|c|c|c|c|c|}
\hline$A_{i}$ & $\mathcal{T} A_{i}$ & $\mathrm{~d}\left(\mathcal{T} A_{0}, \mathcal{T} A_{i}\right)$ & $\alpha\left(A_{0}, A_{i}\right) \vartheta\left(A_{0}, A_{i}\right)$ & $\theta\left(\mathcal{E}\left(A_{0}, A_{i}\right)\right)$ \\
\hline$A_{0}=(4,0)$ & $A_{0}$ & 0 & 0 & 0 \\
$A_{1}=(2 \sqrt{2}, 2 \sqrt{2})$ & $A_{2}$ & 5.656854249 & 5.303300859 & 5.357568053 \\
$A_{2}=(0,4)$ & $A_{3}$ & 7.39103626 & 6.929096494 & 7.628531495 \\
$A_{3}=(-2 \sqrt{2}, 2 \sqrt{2})$ & $A_{4}$ & 8 & 7.5 & 9.145940754 \\
$A_{4}=(-4,0)$ & $A_{5}$ & 7.39103626 & 6.929096494 & 9.678784027 \\
$A_{5}=(-2 \sqrt{2},-2 \sqrt{2})$ & $A_{6}$ & 5.56854249 & 5.303300859 & 9.145940754 \\
$A_{6}=(0,-4)$ & $A_{7}$ & 3.061467459 & 2.870125743 & 7.628531495 \\
$A_{7}=(2 \sqrt{2},-2 \sqrt{2})$ & $A_{0}$ & 0 & 0 & 5.357568053 \\
\hline
\end{tabular}

Therefore, the mapping $\mathcal{T}$ has a unique fixed point, that is $A=(4,0)$.

Corollary 1. Let $(\mathrm{X}, \mathrm{d})$ be a complete metric space and $\mathcal{T}: \mathrm{X} \rightarrow \mathrm{X}$ be a mapping such that

$$
\vartheta(\mathrm{d}(\mathcal{T} \mathrm{x}, \mathcal{T} \mathrm{y})) \leq \theta(\mathcal{E}(\mathrm{x}, \mathrm{y}))
$$

holds for every distinct $\mathrm{x}, \mathrm{y} \in \mathrm{X}$, such that $\mathrm{d}(\mathcal{T} \mathrm{x}, \mathcal{T} \mathrm{y})>0$, where

$$
\mathcal{E}(\mathrm{x}, \mathrm{y})=\max \left\{\mathrm{d}(\mathrm{x}, \mathrm{y})+|\mathrm{d}(\mathrm{x}, \mathcal{T} \mathrm{x})-\mathrm{d}(\mathrm{y}, \mathcal{T} \mathrm{y})|, \frac{\mathrm{d}(\mathrm{x}, \mathcal{T} \mathrm{y})+\mathrm{d}(\mathrm{y}, \mathcal{T} \mathrm{x})}{2}\right\}
$$

and $\vartheta, \theta:(0, \infty) \rightarrow \mathbb{R}$. Suppose that:

$\left(m_{0}\right) \theta(s)<\vartheta(s)$, for all $s>0$;

$\left(m_{1}\right) \vartheta$ lower semi-continuous and non-decreasing;

$\left(m_{2}\right) \lim \sup \theta(s)<\vartheta\left(s_{0}\right)$ for any $s_{0}>0$.

Then, $\mathcal{T}$ possesses a unique fixed point.

Proof. Let $\alpha(\mathrm{x}, \mathrm{y})=1$ in Theorem 3 .

Corollary 2. On a complete metric space $(\mathrm{X}, \mathrm{d})$ let $\mathcal{T}: \mathrm{X} \rightarrow \mathrm{X}$ be a mapping such that

$$
\vartheta(\mathrm{d}(\mathcal{T} \mathrm{x}, \mathcal{T} \mathrm{y})) \leq \kappa \vartheta(\mathcal{E}(\mathrm{x}, \mathrm{y}))
$$

holds for every distinct $\mathrm{x}, \mathrm{y} \in \mathrm{X}$ with $\mathrm{d}(\mathcal{T} \mathrm{x}, \mathcal{T} \mathrm{y})>0$, where $\mathcal{E}(\mathrm{x}, \mathrm{y})$ is defined by $(15), \kappa \in[0,1)$ and $\vartheta:(0, \infty) \rightarrow(0, \infty)$ is left-continuous and non-decreasing. Then $\mathcal{T}$ possesses a unique fixed point.

Proof. Let $\theta(s)=\kappa \vartheta(s)$ in Corollary 1 .

Definition 4. A mapping $\mathcal{T}: \mathrm{X} \rightarrow \mathrm{X}$ on a complete metric space $(\mathrm{X}, \mathrm{d})$ is a $(\alpha, \vartheta, \theta)-\mathcal{E}^{2}-$ contraction if the inequality

$$
\alpha(\mathrm{x}, \mathrm{y}) \vartheta\left(\mathrm{d}\left(\mathcal{T}^{2} \mathrm{x}, \mathcal{T}^{2} \mathrm{y}\right)\right) \leq \theta\left(\mathcal{E}^{2}(\mathrm{x}, \mathrm{y})\right)
$$

holds for every distinct $\mathrm{x}, \mathrm{y} \in \mathrm{X}$, such that $\mathrm{d}\left(\mathcal{T}^{2} \mathrm{x}, \mathcal{T}^{2} \mathrm{y}\right)>0$, where

$$
\mathcal{E}^{2}(\mathrm{x}, \mathrm{y})=\max \left\{\begin{array}{c}
\mathrm{d}(\mathrm{y}, \mathcal{T} \mathrm{y})+|\mathrm{d}(\mathrm{x}, \mathrm{y})-\mathrm{d}(\mathrm{x}, \mathcal{T} \mathrm{x})|, \\
\mathrm{d}(\mathcal{T} \mathrm{x}, \mathcal{T} \mathrm{y})+\left|\mathrm{d}\left(\mathcal{T} \mathrm{x}, \mathcal{T}^{2} \mathrm{x}\right)-\mathrm{d}\left(\mathcal{T} \mathrm{y}, \mathcal{T}^{2} \mathrm{y}\right)\right|, \\
\mathrm{d}\left(\mathcal{T} \mathrm{y}, \mathcal{T}^{2} \mathrm{y}\right)+\left|\mathrm{d}\left(\mathcal{T} \mathrm{x}, \mathcal{T}^{2} \mathrm{x}\right)-\mathrm{d}(\mathrm{y}, \mathcal{T} \mathrm{y})\right|
\end{array}\right\}
$$


Theorem 5. Let $(\mathrm{X}, \mathrm{d})$ be a complete metric space and $\mathcal{T}: \mathrm{X} \rightarrow \mathrm{X}$ be $a(\alpha, \vartheta, \theta)-\mathcal{E}^{2}$ contraction such that

$\left(m_{1}\right) \vartheta$ is lower semi-continuous and non-decreasing;

$\left(m_{2}\right) \limsup _{s \rightarrow s_{0}} \theta(s)<\vartheta\left(s_{0}\right)$ for any $s_{0}>0$;

$\left(m_{3}\right) \mathcal{T}$ is $\alpha$-t.o.a. and there exists $\mathrm{x}_{0} \in \mathrm{X}$, such that $\alpha\left(\mathrm{x}_{0}, \mathcal{T} \mathrm{x}_{0}\right) \geq 1$;

$\left(m_{6}\right) \mathcal{T}^{2}$ is continuous and $\alpha(\mathcal{T} v, v) \geq 1$ for any $v \in\left\{x \in X: \mathcal{T}^{2} \mathrm{x}=\mathrm{x}\right\}$.

Then, $\mathcal{T}$ possesses a fixed point.

Proof. Let $\mathrm{x}_{0} \in \mathrm{X}$ be a point such that $\alpha\left(\mathrm{x}_{0}, \mathcal{T} \mathrm{x}_{0}\right) \geq 1$ and the sequence $\left\{\mathrm{x}_{m}\right\}$ in $\mathrm{X}$, where $\mathrm{x}_{m}=\mathcal{T} \mathrm{x}_{m-1}$, for any $m \in \mathbb{N}$. Since $\mathcal{T}$ is $\alpha$-t.o.a., by Lemma 2 , we get $\alpha\left(\mathrm{x}_{m}, \mathrm{x}_{m+1}\right) \geq 1$, for any $m \in \mathbb{N}$ and then

$$
\begin{aligned}
\vartheta\left(\mathrm{d}\left(\mathrm{x}_{m+2}, \mathrm{x}_{m+3}\right)\right) & \leq \alpha\left(\mathrm{x}_{m}, \mathrm{x}_{m+1}\right) \vartheta\left(\mathrm{d}\left(\mathcal{T}^{2} \mathrm{x}_{m}, \mathcal{T}^{2} \mathrm{x}_{m+1}\right)\right) \\
& \leq \theta\left(\mathcal{E}^{2}\left(\mathrm{x}_{m}, \mathrm{x}_{m+1}\right)\right)<\vartheta\left(\mathcal{E}^{2}\left(\mathrm{x}_{m}, \mathrm{x}_{m+1}\right)\right) .
\end{aligned}
$$

Furthermore, the monotonicity of $\vartheta$ implies that

$$
\mathrm{d}\left(\mathrm{x}_{m+2}, \mathrm{x}_{m+3}\right)<\mathcal{E}^{2}\left(\mathrm{x}_{m}, \mathrm{x}_{m+1}\right),
$$

where

$$
\begin{aligned}
\mathcal{E}^{2}\left(\mathrm{x}_{m}, \mathrm{x}_{m+1}\right)=\max \left\{\begin{array}{c}
\mathrm{d}\left(\mathrm{x}_{m+1}, \mathcal{T} \mathrm{x}_{m+1}\right)+\left|\mathrm{d}\left(\mathrm{x}_{m}, \mathrm{x}_{m+1}\right)-\mathrm{d}\left(\mathrm{x}_{m}, \mathcal{T} \mathrm{x}_{m}\right)\right|, \\
\mathrm{d}\left(\mathcal{T} \mathrm{x}_{m}, \mathcal{T} \mathrm{x}_{m+1}\right)+\left|\mathrm{d}\left(\mathcal{T} \mathrm{x}_{m}, \mathcal{T}^{2} \mathrm{x}_{m}\right)-\mathrm{d}\left(\mathcal{T} \mathrm{x}_{m+1}, \mathcal{T}^{2} \mathrm{x}_{m+1}\right)\right|, \\
\mathrm{d}\left(\mathcal{T} \mathrm{x}_{m+1}, \mathcal{T}^{2} \mathrm{x}_{m+1}\right)+\left|\mathrm{d}\left(\mathcal{T} \mathrm{x}_{m}, \mathcal{T}^{2} \mathrm{x}_{m},\right)-\mathrm{d}\left(\mathrm{x}_{m+1}, \mathcal{T} \mathrm{x}_{m+1}\right)\right|
\end{array}\right\} \\
=\max \left\{\begin{array}{c}
\mathrm{d}\left(\mathrm{x}_{m+1}, \mathrm{x}_{m+2}\right)+\left|\mathrm{d}\left(\mathrm{x}_{m}, \mathrm{x}_{m+1}\right)-\mathrm{d}\left(\mathrm{x}_{m}, \mathrm{x}_{m+1}\right)\right|, \\
\mathrm{d}\left(\mathrm{x}_{m+1}, \mathrm{x}_{m+2}\right)+\left|\mathrm{d}\left(\mathrm{x}_{m+1}, \mathrm{x}_{m+2}\right)-\mathrm{d}\left(\mathrm{x}_{m+2}, \mathrm{x}_{m+3}\right)\right|, \\
\mathrm{d}\left(\mathrm{x}_{m+2}, \mathrm{x}_{m+3}\right)+\mid \mathrm{d}\left(\mathrm{x}_{m+1}, \mathrm{x}_{m+2}\right)-\mathrm{d}\left(\mathrm{x}_{m+1}, \mathrm{x}_{m+2} \mid\right.
\end{array}\right\} \\
=\max \left\{\begin{array}{c}
\mathrm{d}\left(\mathrm{x}_{m+1}, \mathrm{x}_{m+2}\right)+\left|\mathrm{d}\left(\mathrm{x}_{m+1}, \mathrm{x}_{m+2}\right)-\mathrm{d}\left(\mathrm{x}_{m+2}, \mathrm{x}_{m+3}\right)\right|, \\
\mathrm{d}\left(\mathrm{x}_{m+1}, \mathrm{x}_{m+2}\right), \mathrm{d}\left(\mathrm{x}_{m+2}, \mathrm{x}_{m+3}\right)
\end{array}\right\}
\end{aligned}
$$

If for some $m \in \mathbb{N}, \max \left\{\mathrm{d}\left(\mathrm{x}_{m+1}, \mathrm{x}_{m+2}\right), \mathrm{d}\left(\mathrm{x}_{m+2}, \mathrm{x}_{m+3}\right)\right\}=\mathrm{d}\left(\mathrm{x}_{m+2}, \mathrm{x}_{m+3}\right)$ then $\mathcal{E}^{2}\left(\mathrm{x}_{m}, \mathrm{x}_{m+1}\right)=\mathrm{d}\left(\mathrm{x}_{m+2}, \mathrm{x}_{m+3}\right)$ and (20) turns into

$$
\mathrm{d}\left(\mathrm{x}_{m+2}, \mathrm{x}_{m+3}\right)<\mathrm{d}\left(\mathrm{x}_{m+2}, \mathrm{x}_{m+3}\right),
$$

which is a contradiction. If $\max \left\{d\left(x_{m+1}, x_{m+2}\right) d\left(x_{m+2}, x_{m+3}\right)\right\}=d\left(x_{m+1}, x_{m+2}\right)$, then we have $\mathcal{E}^{2}\left(\mathrm{x}_{m}, \mathrm{x}_{m+1}\right)=2 \mathrm{~d}\left(\mathrm{x}_{m+1}, \mathrm{x}_{m+2}\right)-\mathrm{d}\left(\mathrm{x}_{m+2}, \mathrm{x}_{m+3}\right)$ and by (20) we get

$$
\mathrm{d}\left(\mathrm{x}_{m+2}, \mathrm{x}_{m+3}\right)<\mathrm{d}\left(\mathrm{x}_{m+1}, \mathrm{x}_{m+2}\right)
$$

for every $m \in \mathbb{N}$. Therefore,

$$
\mathrm{d}\left(\mathrm{x}_{m+2}, \mathrm{x}_{m+3}\right)<\mathrm{d}\left(\mathrm{x}_{m+1}, \mathrm{x}_{m+2}\right)<\ldots<\mathrm{d}\left(\mathrm{x}_{2}, \mathrm{x}_{3}\right)<\max \left\{\mathrm{d}\left(\mathrm{x}_{1}, \mathrm{x}_{2}\right), \mathrm{d}\left(\mathrm{x}_{0}, \mathrm{x}_{1}\right)\right\} .
$$


Thus, the sequence $\left\{d\left(x_{m}, x_{m+1}\right)\right\}$ is strictly decreasing and bounded below by 0 . Hence, there exist $\delta \geq 0$ such that $\lim _{m \rightarrow \infty} \mathrm{d}\left(\mathrm{x}_{m+1}, \mathrm{x}_{m+2}\right)=\delta$. Suppose that $\delta>0$. Since $\lim _{m \rightarrow \infty} \mathcal{E}^{2}\left(\mathrm{x}_{m}, \mathrm{x}_{m+1}\right)=\lim _{m \rightarrow \infty} \mathrm{d}\left(\mathrm{x}_{m+1}, \mathrm{x}_{m+2}\right)=\delta>0$, letting the limit superior in (19), as $n \rightarrow \infty$ and taking $\left(m_{2}\right)$ into account, we obtain

$$
\vartheta(\delta)=\lim _{m \rightarrow \infty} \vartheta\left(\mathrm{d}\left(\mathrm{x}_{m+2}, \mathrm{x}_{m+3}\right)\right) \leq \limsup _{m \rightarrow \infty} \theta\left(\mathcal{E}^{2}\left(\mathrm{x}_{m}, \mathrm{x}_{m+1}\right)\right)<\limsup _{s \rightarrow \delta} \theta(s)<\vartheta(\delta),
$$

which is a contradiction. Therefore,

$$
\lim _{m \rightarrow \infty} \mathrm{d}\left(\mathrm{x}_{m}, \mathrm{x}_{m+1}\right)=0
$$

and we claim that, in these conditions, the sequence $\left\{x_{m}\right\}$ is Cauchy. If not, from Lemma 1 we can find $e>0$ and two subsequences $\left\{x_{m_{i}}\right\}$ and $\left\{x_{p_{i}}\right\}$ of $\left\{x_{m}\right\}$, such that the equalities in (3), hold. Moreover, we remark that

$$
\begin{aligned}
\mathcal{E}^{2}\left(\mathrm{x}_{m_{i}}, \mathrm{x}_{p_{i}}\right) & =\max \left\{\begin{array}{c}
\mathrm{d}\left(\mathrm{x}_{p_{i}}, \mathcal{T} \mathrm{x}_{p_{i}}\right)+\left|\mathrm{d}\left(\mathrm{x}_{m_{i}}, \mathrm{x}_{p_{i}}\right)-\mathrm{d}\left(\mathrm{x}_{m_{i}}, \mathcal{T} \mathrm{x}_{m_{i}}\right)\right|, \\
\mathrm{d}\left(\mathcal{T} \mathrm{x}_{m_{i}}, \mathcal{T} \mathrm{x}_{p_{i}}\right)+\left|\mathrm{d}\left(\mathcal{T} \mathrm{x}_{m_{i}}, \mathcal{T}^{2} \mathrm{x}_{m_{i}}\right)-\mathrm{d}\left(\mathcal{T} \mathrm{x}_{p_{i}}, \mathcal{T}^{2} \mathrm{x}_{p_{i}}\right)\right|, \\
\mathrm{d}\left(\mathcal{T} \mathrm{x}_{p_{i}}, \mathcal{T}^{2} \mathrm{x}_{p_{i}}\right)+\left|\mathrm{d}\left(\mathcal{T} \mathrm{x}_{m_{i}}, \mathcal{T}^{2} \mathrm{x}_{m_{i}}\right)-\mathrm{d}\left(\mathrm{x}_{p_{i}}, \mathcal{T} \mathrm{x}_{p_{i}}\right)\right|
\end{array}\right\} \\
& =\max \left\{\begin{array}{c}
\mathrm{d}\left(\mathrm{x}_{p_{i}}, \mathrm{x}_{p_{i}+1}\right)+\left|\mathrm{d}\left(\mathrm{x}_{m_{i}}, \mathrm{x}_{p_{i}}\right)-\mathrm{d}\left(\mathrm{x}_{m_{i}}, \mathrm{x}_{m_{i}+1}\right)\right|, \\
\mathrm{d}\left(\mathrm{x}_{m_{i}+1}, \mathrm{x}_{p_{i}+1}\right)+\left|\mathrm{d}\left(\mathrm{x}_{m_{i}+1}, \mathrm{x}_{m_{i}+2}\right)-\mathrm{d}\left(\mathrm{x}_{p_{i}+1}, \mathrm{x}_{p_{i}+2}\right)\right|, \\
\mathrm{d}\left(\mathrm{x}_{p_{i}+1}, \mathrm{x}_{p_{i}+2}\right)+\left|\mathrm{d}\left(\mathrm{x}_{m_{i}+1}, \mathrm{x}_{m_{i}+2}\right)-\mathrm{d}\left(\mathrm{x}_{p_{i}}, \mathrm{x}_{p_{i}+1}\right)\right|
\end{array}\right\}
\end{aligned}
$$

and then, using (21) we get $\lim _{i \rightarrow \infty} \mathcal{E}^{2}\left(\mathrm{x}_{m_{i}}, \mathrm{x}_{p_{i}}\right)=e$. On the other hand, letting $i \rightarrow \infty$ in the below inequality

$$
\begin{gathered}
\mathrm{d}\left(\mathrm{x}_{m_{i}+1}, \mathrm{x}_{p_{i}+1}\right)-\mathrm{d}\left(\mathrm{x}_{m_{i}+1}, \mathrm{x}_{m_{i}+2}\right)-\mathrm{d}\left(\mathrm{x}_{p_{i}+2}, \mathrm{x}_{p_{i}+1}\right) \leq \mathrm{d}\left(\mathrm{x}_{m_{i}+2}, \mathrm{x}_{p_{i}+2}\right) \\
\leq \mathrm{d}\left(\mathrm{x}_{m_{i}+2}, \mathrm{x}_{m_{i}+1}\right)+\mathrm{d}\left(\mathrm{x}_{m_{i}+1}, \mathrm{x}_{p_{i}+1}\right)+\mathrm{d}\left(\mathrm{x}_{p_{i}+1}, \mathrm{x}_{p_{i}+2}\right) .
\end{gathered}
$$

we find that $\lim _{i \rightarrow \infty} \mathrm{d}\left(\mathrm{x}_{m_{i}+2}, \mathrm{x}_{p_{i}+2}\right)=e$. Replacing in (17), $\mathrm{x}$ by $\mathrm{x}_{m_{i}}$ and $\mathrm{y}$ by $\mathrm{x}_{p_{i}}$, and since, by Lemma $2, \alpha\left(\mathrm{x}_{m_{i}}, \mathrm{x}_{p_{i}}\right) \geq 1$, we obtain

$$
\vartheta\left(\mathrm{d}\left(\mathrm{x}_{m_{i}+2}, \mathrm{x}_{p_{i}+2}\right)\right) \leq \theta\left(\mathcal{E}^{2}\left(\mathrm{x}_{m_{i}}, \mathrm{x}_{p_{i}}\right)\right) .
$$

Thus,

$$
\vartheta(e)=\lim _{i \rightarrow \infty} \vartheta\left(\mathrm{d}\left(\mathrm{x}_{m_{i}+2}, \mathrm{x}_{p_{i}+2}\right)\right) \leq \limsup _{i \rightarrow \infty} \theta\left(\mathcal{E}^{2}\left(\mathrm{x}_{m_{i}}, \mathrm{x}_{p_{i}}\right)\right) \leq \limsup _{s \rightarrow e} \theta(s),
$$

which contradicts $\left(m_{2}\right)$. Consequently, $\left\{x_{m}\right\}$ is a Cauchy sequence on a complete metric space, so that, there exists $x_{*}$, such that $\lim _{m \rightarrow \infty} d\left(x_{m}, x_{*}\right)=0$. We claim that $x_{*}$ is a fixed point of $\mathcal{T}$, under the assumptions $\left(m_{6}\right)$.

Now, since $\mathcal{T}^{2}$ is continuous, we have

$$
\lim _{m \rightarrow \infty} \mathrm{d}\left(\mathrm{x}_{m}, \mathcal{T}^{2} \mathrm{x}_{*}\right)=\lim _{m \rightarrow \infty} \mathrm{d}\left(\mathcal{T}^{2} \mathrm{x}_{m-2}, \mathcal{T}^{2} \mathrm{x}_{*}\right)=0,
$$

and then, we derive that $\mathcal{T}^{2} \mathrm{x}_{*}=\mathrm{x}_{*}$. By reductio ad absurdum, we assume that $\mathcal{T} \mathrm{x}_{*} \neq \mathrm{x}_{*}$. Thus, by (17), and keeping in mind $\left(m_{1}\right)$ and $\left(m_{6}\right)$, we have 


$$
\begin{aligned}
& \vartheta\left(\mathrm{d}\left(\mathcal{T} \mathrm{x}_{*}, \mathrm{x}_{*}\right)\right)=\vartheta\left(\mathrm{d}\left(\mathcal{T}^{2}\left(\mathcal{T} \mathrm{x}_{*}\right), \mathcal{T}^{2} \mathrm{x}_{*}\right)\right) \leq \alpha\left(\mathcal{T} \mathrm{x}_{*}, \mathrm{x}_{*}\right) \vartheta\left(\mathrm{d}\left(\mathcal{T}^{2}\left(\mathcal{T} \mathrm{X}_{*}\right), \mathcal{T}^{2} \mathrm{x}_{*}\right)\right) \\
& \leq \theta\left(\mathcal{E}^{2}\left(\mathcal{T} \mathrm{X}_{*}, \mathrm{X}_{*}\right)\right)<\vartheta\left(\mathcal{E}^{2}\left(\mathcal{T} \mathrm{X}_{*}, \mathrm{X}_{*}\right)\right) \\
& =\vartheta\left(\max \left\{\begin{array}{c}
\mathrm{d}\left(\mathrm{x}_{*}, \mathcal{T} \mathrm{x}_{*}\right)+\left|\mathrm{d}\left(\mathcal{T} \mathrm{x}_{*}, \mathrm{x}_{*}\right)-\mathrm{d}\left(\mathcal{T} \mathrm{x}_{*}, \mathcal{T}\left(\mathcal{T} \mathrm{x}_{*}\right)\right)\right|, \\
\mathrm{d}\left(\mathcal{T} \mathrm{x}_{*}, \mathcal{T}\left(\mathcal{T} \mathrm{x}_{*}\right)\right)+\left|\mathrm{d}\left(\mathcal{T}\left(\mathcal{T} \mathrm{X}_{*}\right), \mathcal{T}^{2}\left(\mathcal{T} \mathrm{x}_{*}\right)\right)-\mathrm{d}\left(\mathcal{T} \mathrm{x}_{*}, \mathcal{T}^{2} \mathrm{x}_{*}\right)\right|, \\
\mathrm{d}\left(\mathcal{T} \mathrm{x}_{*}, \mathcal{T}^{2} \mathrm{x}_{*}\right)+\left|\mathrm{d}\left(\mathcal{T}\left(\mathcal{T} \mathrm{x}_{*}\right), \mathcal{T}^{2}\left(\mathcal{T} \mathrm{x}_{*}\right)\right)-\mathrm{d}\left(\mathrm{x}_{*}, \mathcal{T} \mathrm{X}_{*}\right)\right|
\end{array}\right\}\right) \\
& =\vartheta\left(\max \left\{\begin{array}{c}
\mathrm{d}\left(\mathrm{x}_{*}, \mathcal{T} \mathrm{x}_{*}\right)+\left|\mathrm{d}\left(\mathcal{T} \mathrm{x}_{*}, \mathrm{x}_{*}\right)-\mathrm{d}\left(\mathcal{T} \mathrm{x}_{*}, \mathrm{x}_{*}\right)\right| \\
\mathrm{d}\left(\mathcal{T} \mathrm{x}_{*}, \mathrm{x}_{*}\right)+\left|\mathrm{d}\left(\mathrm{x}_{*}, \mathcal{T} \mathrm{x}_{*}\right)-\mathrm{d}\left(\mathcal{T} \mathrm{x}_{*}, \mathrm{x}_{*}\right)\right|, \\
\mathrm{d}\left(\mathcal{T} \mathrm{x}_{*}, \mathrm{x}_{*}\right)+\left|\mathrm{d}\left(\mathrm{x}_{*}, \mathcal{T} \mathrm{x}_{*}\right)-\mathrm{d}\left(\mathrm{x}_{*}, \mathcal{T} \mathrm{x}_{*}\right)\right|
\end{array}\right\}\right) \\
& =\vartheta\left(\mathrm{d}\left(\mathrm{x}_{*}, \mathcal{T} \mathrm{x}_{*}\right)\right),
\end{aligned}
$$

which is a contradiction. Therefore, $\mathrm{d}\left(\mathrm{x}_{*}, \mathcal{T} \mathrm{x}_{*}\right)=0$, so that, $\mathrm{x}_{*}$ is a fixed point of $\mathcal{T}$.

Theorem 6. Adding the condition

$\left(m_{5}\right) \alpha(\mathrm{v}, \mathrm{u}) \geq 1$, for any $\mathrm{v}, \mathrm{u} \in\{\mathrm{x} \in \mathrm{X}: \mathcal{T} \mathrm{x}=\mathrm{x}\}$

to the hypotheses of Theorem 5, one obtains uniqueness of the fixed point.

Proof. In order to prove the uniqueness of the fixed point, we suppose, by reductio ad absurdum, that there exists $\mathrm{x}_{*}, \mathrm{y}_{*} \in\{\mathrm{x} \in \mathrm{X}: \mathcal{T} \mathrm{x}=\mathrm{x}\}$ such that $\mathrm{x}_{*} \neq \mathrm{y}_{*}$. Then, by (17) and $\left(m_{5}\right)$ we obtain

$$
\vartheta\left(\mathrm{d}\left(\mathrm{x}_{*}, \mathrm{y}_{*}\right)\right) \leq \alpha\left(\mathrm{x}_{*}, \mathrm{y}_{*}\right) \vartheta\left(\mathrm{d}\left(\mathcal{T}^{2} \mathrm{x}_{*}, \mathcal{T}^{2} \mathrm{y}_{*}\right)\right) \leq \theta\left(\mathcal{E}^{2}\left(\mathrm{x}_{*}, \mathrm{y}_{*}\right)\right)<\vartheta\left(\mathcal{E}^{2}\left(\mathrm{x}_{*}, \mathrm{y}_{*}\right)\right)=\vartheta\left(\mathrm{d}\left(\mathrm{x}_{*}, \mathrm{y}_{*}\right)\right),
$$

which is a contradiction. Therefore, the mapping $\mathcal{T}$ possesses a unique fixed point.

Remark 2. In case of the mapping $\mathcal{T}$ is continuous, we get the same result without the assumption $\left(m_{6}\right)$.

Example 2. Let the set $\mathrm{X}=[0,5]$ be endowed with the usual metric, $\mathrm{d}: \mathrm{X} \times \mathrm{X} \rightarrow[0,+\infty)$, $\mathrm{d}(\mathrm{x}, \mathrm{y})=|\mathrm{x}-\mathrm{y}|$ and a mapping $\mathcal{T}: \mathrm{X} \rightarrow \mathrm{X}$ defined as $\mathcal{T} \mathrm{x}=\left\{\begin{aligned} 0, & \text { if } \mathrm{x} \in[0,1] \\ 1, & \text { if } \mathrm{x} \in(1,2] \\ \frac{\mathrm{x}-2}{2}, & \text { if } \mathrm{x} \in(2,4] \\ \frac{\mathrm{x}}{2}, & \text { if } \mathrm{x} \in(4,5]\end{aligned}\right.$.

Assume that $\vartheta, \theta:(0, \infty) \rightarrow \mathbb{R}$ are two functions such that $\vartheta$ is non-decreasing and $\theta(s)<$ $\vartheta(s)$, for any $s>0$. First of all, we remark that, taking $\mathrm{x}=4 / 6$ and $\mathrm{y}=7 / 6$, we have

$$
\begin{aligned}
& \mathrm{d}\left(\frac{4}{6}, \frac{7}{6}\right)=\frac{3}{6}, \mathrm{~d}\left(\mathcal{T} \frac{4}{6}, \mathcal{T} \frac{7}{6}\right)=\mathrm{d}(0,1)=1, \mathrm{~d}\left(\frac{7}{6}, \mathcal{T} \frac{7}{6}\right)=\mathrm{d}\left(\frac{7}{6}, 1\right)=\frac{1}{6}, \\
& \mathrm{~d}\left(\frac{4}{6}, \mathcal{T} \frac{4}{6}\right)=\mathrm{d}\left(\frac{4}{6}, 0\right)=\frac{4}{6}, \mathrm{~d}\left(\frac{7}{6}, \mathcal{T} \frac{4}{6}\right)=\mathrm{d}\left(\frac{7}{6}, 0\right)=\frac{7}{6}, \\
& \mathrm{~d}\left(\frac{4}{6}, \mathcal{T} \frac{7}{6}\right)=\mathrm{d}\left(\frac{4}{6}, 1\right)=\frac{2}{6} .
\end{aligned}
$$

Thus,

$$
\vartheta(1)=\vartheta\left(\mathrm{d}\left(\mathcal{T} \frac{4}{6}, \mathcal{T} \frac{7}{6}\right)\right) \leq \theta\left(\mathrm{d}\left(\frac{4}{6}, \frac{7}{6}\right)\right)=\theta\left(\frac{1}{2}\right)<\vartheta\left(\frac{1}{2}\right),
$$

which is a contradiction, so, Theorem 1 can not be applied. 


$$
\begin{aligned}
\vartheta(1) & =\vartheta\left(\mathrm{d}\left(\mathcal{T} \frac{4}{6}, \mathcal{T} \frac{7}{6}\right)\right) \leq \theta\left(\max \left\{\mathrm{d}\left(\frac{4}{6}, \frac{7}{6}\right), \mathrm{d}\left(\frac{7}{6}, \mathrm{~d}\left(\frac{4}{6}, \mathcal{T} \frac{4}{6}\right), \mathcal{T} \frac{7}{6}\right), \frac{\mathrm{d}\left(\frac{7}{6}, \mathcal{T} \frac{4}{6}\right)+\mathrm{d}\left(\frac{4}{6}, \mathcal{T} \frac{7}{6}\right)}{2}\right\}\right) \\
& =\theta\left(\max \left\{\frac{1}{2}, \frac{2}{3}, \frac{1}{6}, \frac{\frac{7}{6}+\frac{2}{6}}{2}\right\}\right)=\theta\left(\frac{3}{4}\right)<\vartheta\left(\frac{3}{4}\right),
\end{aligned}
$$

which is a contradiction. Therefore, Theorem 2 can not be used.

- $\quad$ Since

$$
\mathcal{E}(4 / 6,7 / 6)=\max \left\{\frac{3}{6}+\left|\frac{4}{6}-\frac{1}{6}\right|, \frac{\frac{2}{6}+\frac{7}{6}}{2}\right\}=1,
$$

the inequality (4) becomes

$\vartheta(1) \leq \alpha(4 / 6,7 / 6) \vartheta(\mathrm{d}(\mathcal{T}(4 / 6), \mathcal{T}(7 / 6))) \leq \theta(\mathcal{E}(4 / 6,7 / 6)))<\vartheta(\mathcal{E}(4 / 6,7 / 6))=\vartheta(1)$,

which is again a contradiction (for any function $\alpha: X \times X \rightarrow[0,+\infty]$ ). Thus, Theorem 3 can not be used.

However, we have $\mathcal{T}^{2} x=\left\{\begin{array}{rl}0, & \text { if } x \in[0,4] \\ \frac{x-4}{4}, & \text { if } x \in(4,5]\end{array}\right.$, so that, $\mathcal{T}^{2}$ is continuous. Let $\alpha: X \times$ $X \rightarrow[0,+\infty]$ be the function defined as follows:

$$
\alpha(x, y)=\left\{\begin{aligned}
x^{2}+y^{2}+1, & \text { if } x, y \in[0,4] \\
1, & \text { if } x, y \in(4,5] \\
2, & \text { if } x \in(4,5], y \in[0,1] \\
0, & \text { otherwise. }
\end{aligned}\right.
$$

In this case, it is easy to check that the assumptions $\left(m_{3}\right)$ and $\left(m_{6}\right)$ hold. Let $\vartheta, \theta:(0, \infty) \rightarrow \mathbb{R}$ be two functions such that $\left(m_{1}\right)$ and $\left(m_{2}\right)$ are satisfied, for example $\vartheta(s)=\frac{s}{2}$ and $\theta(s)=\frac{s}{3}$. We show that, the mapping $\mathcal{T}$ is $a(\alpha, \vartheta, \theta)-\mathcal{E}^{2}$ contraction.

1. If $\mathrm{x} \in(4,5]$ and $\mathrm{y} \in[0,1]$, since

$$
\begin{aligned}
& \mathrm{d}(\mathcal{T} \mathrm{x}, \mathcal{T} \mathrm{y})+\left|\mathrm{d}\left(\mathcal{T} \mathrm{x}, \mathcal{T}^{2} \mathrm{x}\right)-\mathrm{d}\left(\mathcal{T} \mathrm{y}, \mathcal{T}^{2} \mathrm{y}\right)\right|=\mathrm{d}\left(\frac{\mathrm{x}}{2}, 0\right)+\mathrm{d}\left(\frac{\mathrm{x}}{2}, \frac{\mathrm{x}-4}{4}\right)=\frac{3 \mathrm{x}+4}{4} \leq \mathcal{E}^{2}(\mathrm{x}, \mathrm{y}), \\
& \mathrm{d}\left(\mathcal{T}^{2} \mathrm{x}, \mathcal{T}^{2} \mathrm{y}\right)=\mathrm{d}\left(\frac{\mathrm{x}-4}{4}, 0\right)=\frac{\mathrm{x}-4}{4},
\end{aligned}
$$

we have

$$
\alpha(\mathrm{x}, \mathrm{y}) \frac{\mathrm{d}\left(\mathcal{T}^{2} \mathrm{x}, \mathcal{T}^{2} \mathrm{y}\right)}{2}=2 \frac{\mathrm{x}-4}{8} \leq \frac{3 \mathrm{x}+4}{12} \leq \frac{\mathcal{E}^{2}(\mathrm{x}, \mathrm{y})}{3}=\theta\left(\mathcal{E}^{2}(\mathrm{x}, \mathrm{y})\right) .
$$

2. If $x, y \in(4,5]$, since

$$
\begin{aligned}
& \mathrm{d}(\mathcal{T} x, \mathcal{T} y)+\left|d\left(\mathcal{T} x, \mathcal{T}^{2} x\right)-d\left(\mathcal{T} y, \mathcal{T}^{2} y\right)\right|=\frac{|x-y|}{2}+\left|\frac{x+4}{4}-\frac{y+4}{4}\right| \\
& \quad=\frac{3|x-y|}{2} \leq \mathcal{E}^{2}(x, y), \\
& d\left(\mathcal{T}^{2} x, \mathcal{T}^{2} y\right)=d\left(\frac{x-4}{4}, \frac{y-4}{4}\right)=\frac{|x-y|}{4},
\end{aligned}
$$

we get

$$
\alpha(\mathrm{x}, \mathrm{y}) \vartheta\left(\mathrm{d}\left(\mathcal{T}^{2} \mathrm{x}, \mathcal{T}^{2} \mathrm{y}\right)\right)=\frac{|\mathrm{x}-\mathrm{y}|}{8} \leq \frac{3|\mathrm{x}-\mathrm{y}|}{6} \leq \frac{\mathcal{E}^{2}(\mathrm{x}, \mathrm{y})}{3}=\theta\left(\mathcal{E}^{2}(\mathrm{x}, \mathrm{y})\right) .
$$

Therefore, by Theorem 5, the mapping $\mathcal{T}$ has a unique fixed point, this being $\mathrm{x}=0$. 
Corollary 3. Let $(\mathrm{X}, \mathrm{d})$ be a complete metric space and $\mathcal{T}: \mathrm{X} \rightarrow \mathrm{X}$ be a mapping, such that

$$
\vartheta\left(\mathrm{d}\left(\mathcal{T}^{2} \mathrm{x}, \mathcal{T}^{2} \mathrm{y}\right)\right) \leq \theta\left(\mathcal{E}^{2}(\mathrm{x}, \mathrm{y})\right)
$$

holds for every distinct $\mathrm{x}, \mathrm{y} \in \mathrm{X}$ with $\mathrm{d}\left(\mathcal{T}^{2} \mathrm{x}, \mathcal{T}^{2} \mathrm{y}\right)>0$, where

$$
\mathcal{E}^{2}(\mathrm{x}, \mathrm{y})=\max \left\{\begin{array}{c}
\mathrm{d}(\mathrm{y}, \mathcal{T} \mathrm{y})+|\mathrm{d}(\mathrm{x}, \mathrm{y})-\mathrm{d}(\mathrm{x}, \mathcal{T} \mathrm{x})| \\
\mathrm{d}(\mathcal{T} \mathrm{x}, \mathcal{T} \mathrm{y})+\left|\mathrm{d}\left(\mathcal{T} \mathrm{x}, \mathcal{T}^{2} \mathrm{x}\right)-\mathrm{d}\left(\mathcal{T} \mathrm{y}, \mathcal{T}^{2} \mathrm{y}\right)\right|, \\
\mathrm{d}\left(\mathcal{T} \mathrm{y}, \mathcal{T}^{2} \mathrm{y}\right)+\left|\mathrm{d}\left(\mathcal{T} \mathrm{x}, \mathcal{T}^{2} \mathrm{x}\right)-\mathrm{d}(\mathrm{y}, \mathcal{T} \mathrm{y})\right|
\end{array}\right\},
$$

and $\vartheta, \theta:(0, \infty) \rightarrow \mathbb{R}$. Suppose that:

$\left(m_{0}\right) \theta(s)<\vartheta(s)$, for all $s>0$;

$\left(m_{1}\right) \vartheta$ is lower semi-continuous and non-decreasing;

$\left(m_{2}\right) \lim \sup \theta(s)<\vartheta\left(s_{0}\right)$ for any $s_{0}>0$;

$\left(m_{6}\right) \mathcal{T}^{2}$ is continuous.

Then, $\mathcal{T}$ possesses a unique fixed point.

Proof. Let $\alpha(\mathrm{x}, \mathrm{y})=1$ in Theorem 5 .

Corollary 4. On a complete metric space $(\mathrm{X}, \mathrm{d})$ let $\mathcal{T}: \mathrm{X} \rightarrow \mathrm{X}$ be a mapping, such that

$$
\vartheta\left(\mathrm{d}\left(\mathcal{T}^{2} \mathrm{x}, \mathcal{T}^{2} \mathrm{y}\right)\right) \leq \kappa \vartheta\left(\mathcal{E}^{2}(\mathrm{x}, \mathrm{y})\right)
$$

holds for every distinct $\mathrm{x}, \mathrm{y} \in \mathrm{X}$ with $\mathrm{d}\left(\mathcal{T}^{2} \mathrm{x}, \mathcal{T}^{2} \mathrm{y}\right)>0$, where

$$
\mathcal{E}^{2}(\mathrm{x}, \mathrm{y})=\max \left\{\begin{array}{c}
\mathrm{d}(\mathrm{y}, \mathcal{T} \mathrm{y})+|\mathrm{d}(\mathrm{x}, \mathrm{y})-\mathrm{d}(\mathrm{x}, \mathcal{T} \mathrm{x})| \\
\mathrm{d}(\mathcal{T} \mathrm{x}, \mathcal{T} \mathrm{y})+\left|\mathrm{d}\left(\mathcal{T} \mathrm{x}, \mathcal{T}^{2} \mathrm{x}\right)-\mathrm{d}\left(\mathcal{T} \mathrm{y}, \mathcal{T}^{2} \mathrm{y}\right)\right|, \\
\mathrm{d}\left(\mathcal{T} \mathrm{y}, \mathcal{T}^{2} \mathrm{y}\right)+\left|\mathrm{d}\left(\mathcal{T} \mathrm{x}, \mathcal{T}^{2} \mathrm{x}\right)-\mathrm{d}(\mathrm{y}, \mathcal{T} \mathrm{y})\right|
\end{array}\right\}
$$

$\kappa$ in $(0,1)$ and $\vartheta:(0, \infty) \rightarrow(0, \infty)$ is left-continuous and non-decreasing. If $\mathcal{T}^{2}$ is continuous, then $\mathcal{T}$ possesses a unique fixed point.

Proof. Let $\theta(s)=\kappa \vartheta(s)$ in Corollary 3 .

Remark 3. We mention that a series of known results can be found through a convenient choices of functions $\alpha, \vartheta$ and $\theta$. For example, by choosing $\alpha(\mathrm{x}, \mathrm{y})=1, \vartheta(\mathrm{s})=\mathrm{s}$ and $\theta(\mathrm{s})=\beta(\mathrm{s}) \mathrm{s}$, where $\beta:(0, \infty) \rightarrow(0,1)$ is such that $\limsup _{s \rightarrow e} \beta(s)<1$ for any $e>0$, Theorem 3 reduces to the main Theorem in [1].

Author Contributions: Writing-review and editing: M.A.A., S.G.-O., A.F. All authors contributed equally and significantly in writing this article. All authors have read and agreed to the published version of the manuscript.

Funding: We declare that funding is not applicable for our paper.

Data Availability Statement: No data were used to support this study.

Acknowledgments: The authors thanks anonymous referees for their remarkable comments, suggestions, and ideas that help to improve this paper.

Conflicts of Interest: The authors declare that they have no competing interests. 


\section{References}

1. Fulga, A.; Proca, A. A new Generalization of Wardowski Fixed Point Theorem in Complete Metric Spaces. Adv. Theory Nonlinear Anal. Its Appl. 2017, 1, 57-63. [CrossRef]

2. Alqahtani, B.; Fulga, A.; Karapinar, E. A short note on the common fixed points of the Geraghty contraction of type $E_{S, T}$. Demonstr. Math. 2018, 51, 233-240. [CrossRef]

3. Fulga, A.; Karapınar, E. Revisiting of some outstanding metric fixed point theorems via E-contraction. Analele Univ. OvidiusConstanta-Ser. Mat. 2018, 26, 73-97. [CrossRef]

4. Karapınar, E.; Fulga, A.; Aydi, H. Study on Pata E-contractions. Adv. Differ. Equ. 2020, 2020, 539. doi:10.1186/s13662-020-02992-4 [CrossRef]

5. Proinov, P. Fixed point theorems for generalized contractive mappings in metric spaces. J. Fixed Point Theory Appl. 2020, $22,21$. [CrossRef]

6. Karapinar, E.; Samet, B. Generalized $(\alpha-\psi)$ contractive type mappings and related fixed point theorems with applications. Abstr. Appl. Anal. 2012, 2012, 793486. [CrossRef]

7. Alsulami, H.; Gulyaz-Ozyurt, S.; Karapınar, E.; Erhan, I.M. Fixed point theorems for a class of alpha-admissible contractions and applications to boundary value problem. Abstr. Appl. Anal. 2014, 2014, 187031. [CrossRef]

8. Aydi, H.; Karapınar, E.; Zhang, D. A note on generalized admissible-Meir-Keeler-contractions in the context of generalized metric spaces. Results Math. 2017, 71, 73-92. [CrossRef]

9. Aksoy, U.; Karapınar, E.; Erhan, I.M. Fixed points of generalized alpha-admissible contractions on b-metric spaces with an application to boundary value problems. J. Nonlinear Convex Anal. 2016, 17, 1095-1108.

10. Arshad, M.; Ameer, E.; Karapınar, E. Generalized contractions with triangular alpha-orbital admissible mapping on Branciari metric spaces. J. Inequalities Appl. 2016, 2016, 63. [CrossRef]

11. Karapınar, E.; Sen, M.D.L.; Fulga, A. A Note on the Górnicki-Proinov Type Contraction. J. Funct. Spaces 2021, $2021,6686644$.

12. Karapınar, E.; Fulga, A. A fixed point theorem for Proinov mappings with a contractive iterate. Appl. Math. J. Chin. Univ. in press.

13. Popescu, O. Some new fixed point theorems for $\alpha$-Geraghty contraction type maps in metric spaces. Fixed Point Theory Appl. 2014, 2014, 190. [CrossRef] 